\title{
Real Time Monitoring of Solar PV Parameter Using IoT
}

\author{
Shailesh Sarswat, Indresh Yadav, Sanjay Kumar Maurya
}

\begin{abstract}
This paper presents a system design to monitor real-time Solar Photovoltaic System (SPV) parameters using the Internet of Thing (IoT) technology. Some essential parameters of an SPV system such as Voltage, Current, and panel temperature is being sensed using sensors. While the most crucial parameter power of the SPV is computed. These parameters are transferred over the cloud with the help of node MCU Esp8266. An android application fetches the cloud data. For this, a complete application is developed on android studio for mobile application for real-time monitoring the PV panel output Voltage, Current, Power and Temperature. The system is tested in the laboratory to monitor the SPV parameter.
\end{abstract}

Keywords: Solar Photovoltaic System, IoT, Voltage Sensor, Current Sensor, Parameter Monitoring, Android Apk.

\section{INTRODUCTION}

$\mathrm{N}$ ow, these days every human being needs electrical power for their quality of life. The consumption of power is increasing everyday lives, and at the same time, other energy sources are depreciating day by day. Therefore, to meet the demand for power, other sources of power is required. For the Generation of Electricity, there can be two ways first one is from Non-Renewable Sources, and another one is Renewable Sources. Non-renewable sources are not regenerated by nature after first use such Fossil Fuels, Coal, Natural Gas, Nuclear Fuel while Renewable Sources can be utilized again and again which is not depreciating never such as sun, Geothermal, Wind Energy and Tidal Energy. Solar power is, therefore, is said to be a sustainable power source [1].

Solar power has become more popular in the world as it is available in plenty amount with minimal impact on the environment. Though, the solar power generation becomes cheaper with advances in the conversion technology. To make the solar system accessible the monitoring at the customer level is the need of present [2]-[3]. More suitable sources of energy and in few upcoming years, may overcome non- renewable energy sources completely. Solar PV system consistently generating enough amount of power. Hence

Revised Manuscript Received on November 27, 2019.

* Correspondence Author

Shailesh Sarswat, Electrical Engineering department, GLA University, Mathura, India.

Email:sarswat.arvindnath_mtee17@gla.ac.in saraswatshailesh@gmail.com

Indresh Yadav, Electrical Engineering Department, GLA University, Mathura, India. Email: indresh.yadav@gla.ac.in

Dr. Sanjay Maurya, Electrical Engineering Department, GLA University, Mathura, India Email: sanjay.maurya@gla.ac.in

there is a need to track its performance in real time [4]. The real-time monitoring of solar parameter finds the solution on the Internet of Thing Technology. An IoT is a device which can communicate the between the machine and the Cloud. The use of IoT enabled us to fetch the data from cloud wirelessly. Monitoring of system parameters is vital in any solar power generation system. The important solar photovoltaic system parameter of i.e. current, voltage and temperature is sensed by various sensors real-time monitoring [5]. A Remote monitoring of Solar Photovoltaic systems has many challenges such as it has to deals with, tracking the panel voltage, current and temperature and Real data of the solar system is to be synchronized time to time. For this node mcu is used which communicates between the machines and transfer the data over think speak cloud. The proposed system has flexibility by using hi-tech GPRS to connect the Solar PV to a cloud server [6].

\section{PROPOSED SYSTEM}

In the Prototype system, Node Mcu Esp8266 is used here to interfacing with solar panel and sensors. Panel voltage is obtained by applying in voltage sensor in voltage divider circuit. The current is sensed by current sensing circuit and temperature by temperature sensor. All the data is then transmitted to remote server with the help of node mcu which is Wi-Fi gateway transfer the data to cloud. The cloud data is retrieved by user using mobile application.

\section{A. Proposed methodology}

The proposed system for monitoring the solar module using IoT is shown in Fig.1. The parameters voltage, current and temperature are monitored by using the sensor mounted on PV panel and Power Conditioning Units (PCU). Power is obtained by using mathematical formulation.

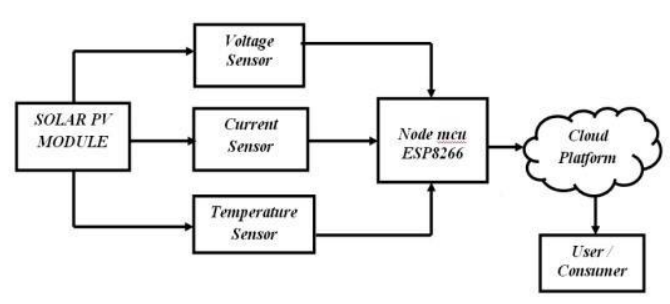

Fig. 1. Block Diagram of Solar PV Monitoring System 


\section{Real Time Monitoring of Solar PV Parameter Using IoT}

For sensing the voltage voltage sensor is In the methodology, we can see that the power flow of the model is explained in that the solar radiance energy i.e. sunlight from the source sun is trapped by solar panels the solar panel converts the solar energy into electrical energy. This electrical energy is then sensed by various sensors such as voltage generated by solar panel is sensed by voltage sensor for measuring voltage with the help of voltage divider principle and current produced by solar panel is measured by current sensor module and temperature or heat energy available or fall on solar panel is tracked by the temperature sensor.

All collected data of voltage, current and temperature sensor is then fed to Node MCU which converts the signals into digital using serial interface and mcu act as a gateway and sends this data over the cloud server and then this data is accessed via user over the application.

\section{B. Communication Pattern}

The communication pattern for IoT device is shown in below fig 2 . here we can see that communication can be done forwarded either by cloud platform or by his/her smartphones with the help of the mobile application.

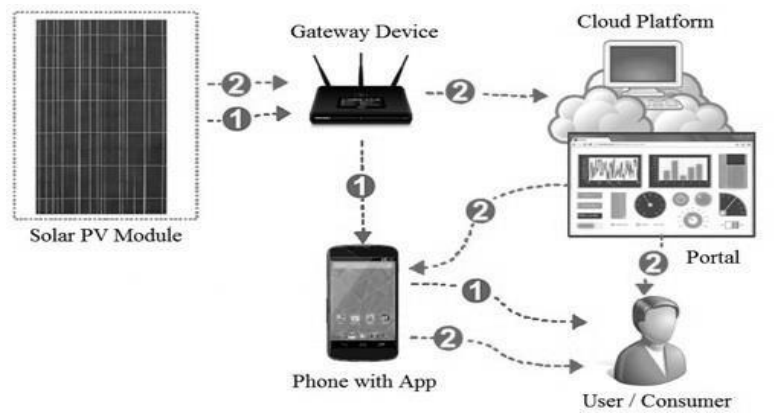

Fig. 2. Communication Pattern [8]

\section{SOFTWARE}

It includes the programming of the Esp8266. The programming language used for the Esp8266 is in c level programming language as it easy to program in Arduino Ide or Esplora.

The data which send over the cloud can be accessed by app therefore to read that data proper login is to required therefore a channel is made to read the data on the application.

\section{A. Android App Studio}

Android app Studio is an integrated development Environment for making application of android mobile phones it which comes into the market on 16 may 2013. Using this software application for android mobile phone can be made in simple and in ease manner. The development language for app making can be java, c, c++ etc.

\section{B. Think Speak Cloud}

Think speak is used as an open source IOT Platform, it accesses the data from the sensors and stores it or it captures the data of the things which is connected to the systems through internet using HTTP Protocol Provided by the local in two ways viz. firstly the information to the user can be

network to the cloud. It synchronizes all the data records received from the sensors, and send updates to user via things speak application. To utilize this consumer, have to make an account on things speak clouds that contains various channels for various parameter of monitoring system in a remote device. It provides the admin or the consumer to read and visualize the data in graphical representation. Due to this user can read the data from anywhere from any place.

Thinkview application in which just by click on the add channel icon to make their channel it can be public or private in public mode the data is seen by anyone just by adding their channel no. while in private model it requires a key to read the data or write the data of application of IOT devices[11].

\section{HARDWARE}

Fig. 3. Solar Panel Monitoring Installed App

\section{A. Solar Panel}

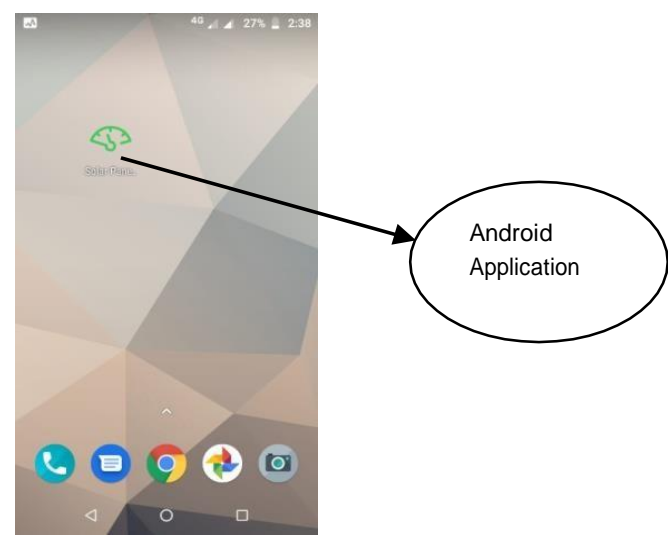

The Solar panel is a device which is used to transfer the solar energy into electrical energy by absorbing the sunlight. There are different types of solar panels and classified in various types but we opt for polycrystalline solar panel as it can work in even winter seasons where it can generate electricity in daylight too and have medium efficiency between $15-17 \%$ and at same time its is cheaper then monocrystalline and operating temperature range is between -40 to 85 degree celsius.

\section{B. Node Mcu Esp8266}

It is a Wi-Fi soc chip which provides open IOT open source platform since the platform is open source design is open for edit and modify. This Wi-Fi chip is a low cost development kit developed by Express if system having TCPIIP protocol. We used version 2 Node Mcu. Serial communication protocol is used by it like UART, SPI, I2C etc which is used to connect various devices LCD display, GPS Module etc

\section{Sensor}

Sensor is a device or module which can detect the cha nge in the electronic devices and convert the physical quantity into an electrical signal and send this signal to other electronic device who is capable of reading this data. The various sensors used in system design is discussed ahead. 


\section{- Voltage Sensor}

Sensor which is used to measure the solar panel voltage is voltage sensor module, we uses the voltage sensor module which can measure voltage up to $25 \mathrm{~V}$. its works on the principle of voltage divider principle [5],[12]

$$
V_{\text {out }}=\frac{R_{2}}{R_{1}+R_{2}} V_{\text {in }}
$$

Where,

$\mathrm{V}_{\text {out }}=$ Output voltage of the sensor module,

$\mathrm{V}_{\text {in }}=$ Input voltage of the sensor module,

$\mathrm{R}_{1}=$ input to where voltage is to be measured \&

$\mathrm{R}_{2}=$ parallel to measuring device and input to node MCU

\section{- Current Sensor}

Sensor which is used to track the solar panel current is current Sensor, its works on the principle of hall effect sensor and measure the current. Current sensor we are using is ACS712 which is capable of measuring current up to $20 \mathrm{~A}$

\section{- Temperature Sensor}

Temperature sensor measures the temperature of the solar panel as we know the power generated by solar panel is directly related to temperature at the panel i.e if temperature is high at panel then the voltage current generated is also more or vice versa. Therefore, measurement of temperature is necessary which is done by temperature sensor module dht11[12].

\section{EXPERIMENTAL SETUP}

The Hardware Arrangement of the prototype model is shown in Fig 4a, 4b below figure here we can see that two Esp8266 is used one for current sensing while other for voltage Sensing and temperature sensor Dht11 is also connected to Node MCU Esp8266 which sense the temperature or radiating receiving at solar module. Power to model is given by Ac to Dc converter as Esp8266 required 5V DC input.

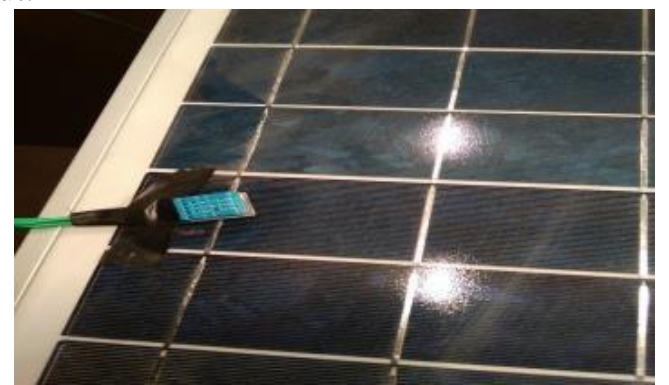

Fig4a. Temperature Sensor Dht11 Installed on Solar Panel

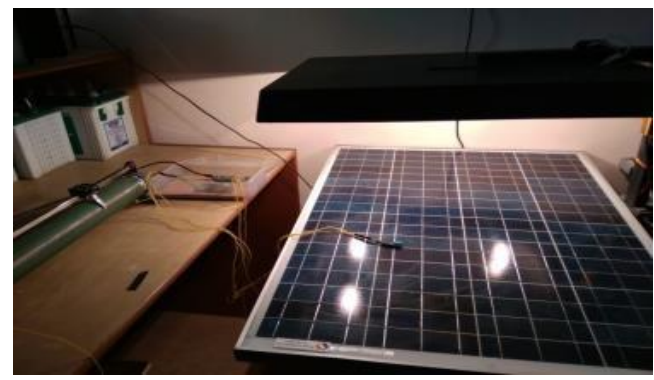

Fig4b. Hardware Model of Solar monitoring System Using IOT
Fig4c. shows the flow Chart of communication with IoT. When the data is obtained from solar PV PCU the connection is initialized in Embedded system gate way. Now the embedded system gateway collects the data from Solar PV PCU and stored in temporary buffers. In embedded system gateway we used is Esp8266 Node MCU Programming is written using the Arduino IDE using $\mathrm{C}++$.

When all solar panel parameters are received from the Solar PV System, then embedded system gateway begins to send the data serially over the app. The following figure Explains the working of the IOT system for monitoring the

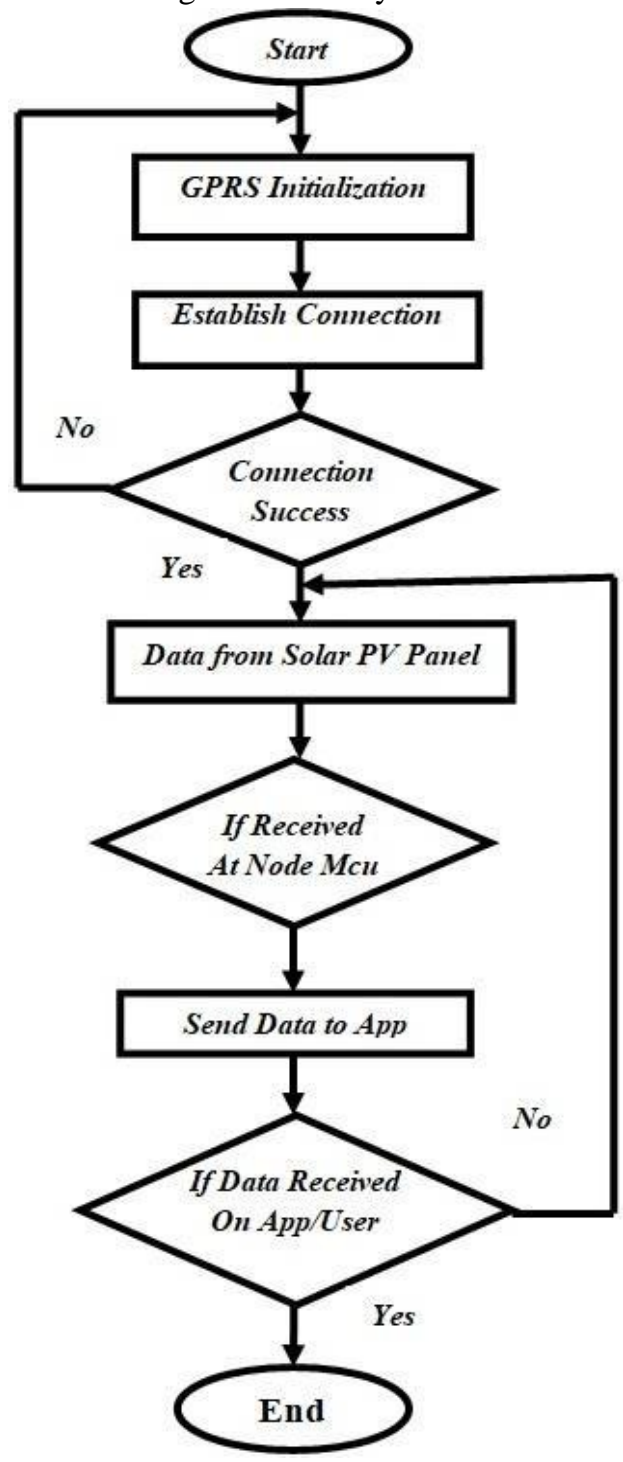

Fig 4c. Flow Chart of communication with IOT

solar PV.

The data can be viewed at anytime and anywhere and also it provides the real time status of solar PV Panel in regular interval of time in previous papers they all have used lora, arduino uno but in this research we avoid the use of any arduino uno, raspberry pi and lora system so the overall system is became more affordable i.e. the cost of the overall system is reduced as that of previous techniques. 


\section{RESULTS}

The following results are obtained on the mobile application by various sensors and various plots of voltage, current, temperature and power plots are made in the mobile application interface and value of sensor is refreshed in every one minute interval and new value is recorded by the IOT device.

\section{A. Voltage Plot}

This plot showing the 44reading which observed by voltage sensor during the day time between $12.27 \mathrm{pm}$ and $12.52 \mathrm{pm}$ and the maximum values sense or generated by solar PV Panel is $23.64 \mathrm{~V}$ while minimum value is $20.88 \mathrm{~V}$ as shwon in fig 5 a.

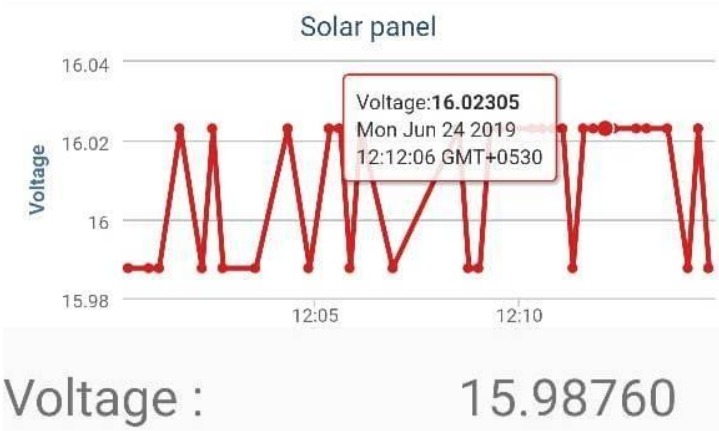

Fig5a. Voltage Measurement by Voltage Sensor

\section{B. Current Plot}

It's showing the value of Current across the load i.e shunt Resistance which is recorded by current sensor ACS712 during time between $12.27 \mathrm{pm}$ and $12.52 \mathrm{pm}$ and the maximum current sensed is 0.78 Amps while minimum value is $0.58 \mathrm{Amps}$ as shown in Fig. 5 b.

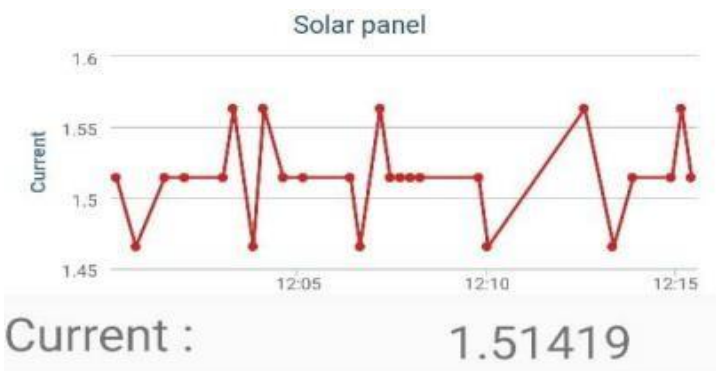

Fig 5b. Current Plot by Current Sensor ACS712

\section{Temperature Plot}

Dht11 is used to sense the temperature of the solar panel surface i.e it sense the radiance received on it by the sun during the day time in degree Celsius. Plot of temperature sensor is given below the maximum temperature reached is 40.90 degree Celsius and minimum temperature reached is 37.50 degree Celsius as shown in fig $5 \mathrm{c}$.

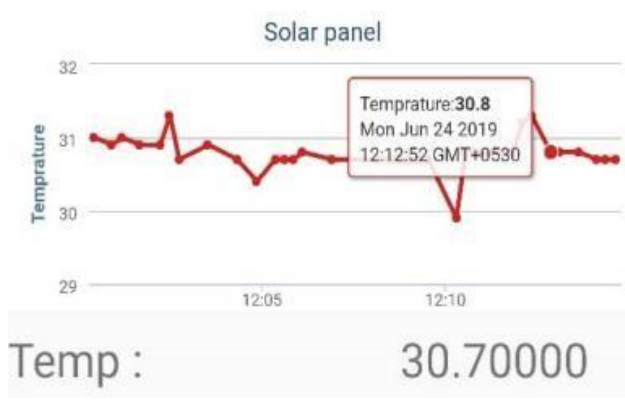

Fig5c. Panel Temperature

\section{Power Plot}

Power curve is the product of both current and voltage as from formulae $\mathrm{P}=\mathrm{V} \times \mathrm{I}$. The following power Curve is obtained from the model on the application interface. Fig $5 d$ shows the power output from the panel.

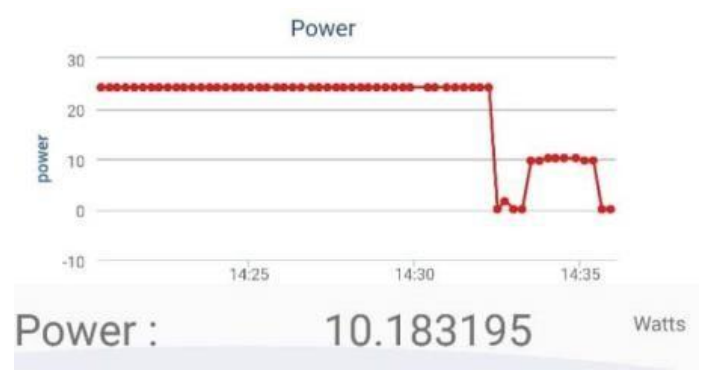

Fig5d. Power Output.

\section{CONCLUSION}

An IoT Application of remote monitoring System of Solar PV using node Mcu has been tested practically and proven to work adequately by monitoring the parame- ters successfully. The node mcu is programmed in c language in Esplora. The IoT Application is designed on the software Android App Studio which is capable to monitoring voltage current and temperature and power. The data is transmitted by node mcu and received on the solar panel monitoring application by using internet The system is tested on single panel and it can be expanded to with more no. panels and it is off grid system can be upgrade on grid system in future frame work.

\section{REFERENCES}

1. G.Subhasri, “A Study of IoT based Solar Panel Tracking System,” vol. 11, no. 7, pp. 537- 545, 2018.

2. S. Patil, M. Vijayalashmi, and R. Tapaskar, "Solar Energy Monitoring Ssystem Using IoT," vol. 15, no. 2, pp. 149-155, 2017.

3. A. Kekre, "Solar Photovoltaic Remote Monitoring System Using IOT," pp. 27-29, 2017.

4. P.A. Patil, "GRID TIE Solar Power Plant Data Acquisition System using Internet of Things,” 2018 Int. Conf. Inf., Commun. Eng. Technol., pp. 1-4 2018.

5. S.Suryono, "Wireless Sensor System for Photovoltaic Panel Efficiency Monitoring Using Wi-Fi Network.

6. R. F. Gusa, "Monitoring System for Solar Panel Using Smartphone Based on Monitoring System for Solar Panel Using Smartphone Based on Microcontroller," no. April, 2018.

7. B. Shrihariprasath and V. Rathinasabapathy, "A smart IoT system for monitoring solar PV power conditioning unit," IEEE WCTFTR 2016 Proc. 2016 World Conf. Futur. Trends Res. Innov. Soc. Welf., 2016.

8. C. Perera, C. H. Liu, and S. Jayawardena, "IEEE TRANSACTIONS ON EMERGING TOPICS IN COMPUTING The Emerging Internet of Things Marketplace From an Industrial Perspective: A Survey," vol. 3, no. 4, 2015. 
9. H. R. Esmaeel, “Apply Android Studio ( SDK ) Tools,” vol. 5, no. 5, pp. 88-93, 2015.

10. N. Verma, S. Kansal, and H. Malvi, "Development of Native Mobile Application Using Android Studio for Cabs and Some Glimpse of Cross Platform Apps," vol. 13, no. 16, pp. 12527-12530, 2018.

11. R. L. R. L. Babu, D. Rambabu, A. R. Naidu, R. D. Prasad, and P. G. Krishna, "IoT Enabled Solar Power Monitoring System," vol. 7, pp. 526-530, 2018

12. S.Badave, M. Sagar, B. Ranjana, and P. Sanjeev Kumar, "Health Monitoring System of Solar Photovoltaic Panel: An Internet of Things Health Monitoring System of Solar Photovoltaic Panel: An Internet of Things Application,” no. November 2017, 2016.

\section{AUTHORS PROFILE}

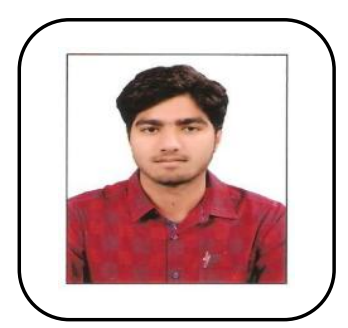

Shailesh Sarswat has earned his Bachelor in Engineering and M.Tech. both in Electrical Engineering from The Rashtrasant Tukdogi Maharaj Nagpur University, Nagpur (India) and GLA University, Mathura (India) respectively. His research interest includes Power Electronics Devices and solar PV optimizations.

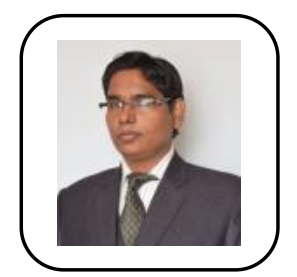

Indresh Yadav has earned his B.Tech. in Electrical Engineering (with First-Class) from G.B.T.U. (now A. K. T.U.) Lucknow, Uttar Pradesh, India, in 2010 and his M.Tech. in, Power Electronics and Drives, Electrical Engineering (with First-Class) from K.N.I.T. Sultanpur, Uttar Pradesh, India, in Feb 2013. Now he is pursuing $\mathrm{Ph.D.}$. in Renewable Energy from Institute of Engineering and Technology, GLA University Mathura, Uttar Pradesh, India. His research interests include the area of energy efficient control and application of power electronics on machine and renewable energy sources. He is a member of IET.

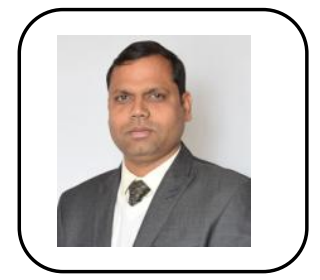

Sanjay Kumar Maurya has received his Bachelor Degree from Dayalbagh Educational Institute, Agra, in 1998. And he has earned his M.Tech. \&Ph.D. from Motilal Nehru National Institute of Technology, Allahabad. His research Interests includes Image processing, Electric Vehicle and Solar Power Generation. He has supervised Six Master Dissertation and supervising three Research Scholars. The research Scholars working under his supervision is working on Solar Power, Electric Vehicle. He has published many research papers in Referred Journal and International Proceeding. He is senior member IEEE, ICEIT and student advisor in IEEE PES student chapter in GLA University. 